\title{
Mushroom Poisoning-an overview
}

\author{
B. S. Patowary \\ Professor, Department of Medicine, College of Medical Sciences, Bharatpur, Nepal
}

\begin{abstract}
There are many thousands of mushroom species in the world, some are edible and some are poisonous due to containing significant toxins. The edible mushroom is a common food item with tempting flavour, taste and nutritive value; nowadays quite often grown at home and cultured with commercial marketing. Mushroom poisoning usually results from ingestion of wild mushrooms due to misidentification of a toxic mushroom as an edible species bearing very close resemblance, deliberate seeking of psychotropic mushrooms and accidental childhood ingestions. Majority of fatal mushroom poisoning occurs due to ingestion of Amanita Phalloides - the 'death cap', due to its high content of Amatoxin - a potent cytotoxin. Fatal poisoning is usually associated with delayed onset of symptoms which are very severe, with hepatic, renal, hemolytic and CNS involvement. Aim of this article is for informational and preventive purpose.
\end{abstract}

Key words: Mushroom poisoning, amanita phalloidis, amatoxin.

Mushroom poisoning refers to deleterious effects from ingestion of toxic substances present in a mushroom. The toxins present are secondary metabolites produced in specific biochemical pathways in the fungal cell. Majority of fatal poisoning worldwide are attributable to the Amanita Phalloides mushroom - appropriately named "death cap"; other dangerous species of mushrooms are Amanita verna, Amanita virosa , Gyromitra, Gallerina and Lepiota species - all of which contain amatoxin, a potent cytotoxin. Ingestion of even a portion of one mushroom of a dangerous species may be sufficient to cause death. The lethal dose of Amatoxin is $\sim 10 \mathrm{mg}$, the amount found in a single death cap mushroom. ${ }^{1}$

Correspondences: Dr. B. S. Patowary

E-mail: drbspatowary@yahoo.com
The characteristic pathologic finding in fatalities from amatoxin containing mushroom poisoning is acute massive necrosis of liver parenchyma. ${ }^{1}$ Hemolysis and renal failure are common accompaniments with occasional pancreatitis.

Mushroom poisoning is usually the result of ingestion of wild mushrooms due to misidentification of a toxic mushroom as an edible species bearing close resemblance. It is also found as a result of small children, specially toddlers in the 'grazing' stage ingesting mushrooms found in the lawn. "Magic" mushrooms are intentionally taken by adolescents for their hallucinogenic effects. ${ }^{2}$

The clinical manifestations of mushroom poisoning depend mainly on the type of mushroom involved (the degree of toxic species), amount ingested (obvious), age (symptoms in children are more fatal compared to 
B. S. Patowary, Mushroom Poisoning-an overview

an adult due to their having low body weight), time of onset of symptoms (very toxic mushroom poisoning cases usually have delayed onset of symptoms which are very severe with hepatic, renal, hemolytic and CNS involvement), geographic distribution (some of the dangerous species of mushrooms could be more abundant in some locations; also some specimen that look edible at one geographic location may be deadly in another region) as well as premorbid hepatic and renal condition.

\section{Toxins and their manifestations}

The clinical manifestations of mushroom poisoning are dependent on the fungal species involved. These may range from minor gastro - intestinal disturbances to hallucinations, delirium, cramps and even fatal consequences.

The highest reported incidences of mushroom poisoning and fatality are due to consumption of Amanita Phalloides. This mushroom produces different toxins. $^{3}$

1.Amatoxin (alpha, beta and gamma amanitins ) - a thermostable nitrogenous bicyclic octapeptide insoluble in water. It irreversibly binds to and inhibits RNA polymerase II, blocking the production of DNA , the basis of cell reproduction; this leads to the death of many cells specially those that reproduce frequently e.g liver, intestine, kidneys and ultimately the CNS.

It binds to the muscle protein actin - essential for muscle contraction, thus delaying the ability of certain muscle groups to contract, resulting in weakness.

It produces acute liver failure, producing extensive parenchymal necrosis as well as renal failure and occasionally pancreatic toxicity. It is eight fold more toxic than Phallotoxin.
Kinetics of amatoxin - it rapidly disappears from plasma, excreted in urine during first few days following ingestion; large amount may be eliminated in faeces.

1. Phallotoxin - also a hepatic toxin and gastro intestinal irritant.

2.Amanita haemolysin - a hemolytic glycoside that breaks down RBCs.

Amatoxin is also present in other mushrooms ${ }^{3}$ viz. A verna, A virosa and Galerina. Lapiota species is highly toxic and is associated with a fatality rate of about $20 \%{ }^{4}$ and in children, it is higher.

\section{Clinical manifestations of amatoxin poisoning}

They are briefed as this is the most fatal toxin.

The manifestations occur in different stages or phases. ${ }^{4}$ These include:

1. A characteristic latent period of 6-24 hours post ingestion before onset of symptoms.

2. Then abdominal cramping, nausea, vomiting and severe watery diarrhoea occur that usually

Last for 24 hours; fluid losses may be severe enough to cause profound dehydration, even circulatory collapse.

3. A period of remission of symptoms occurs that last 1-3 days (if hospitalised, the patient is sometimes released).However on going liver damage is occurring as indicated by laboratory investigations (elevation of serum amino transferase levels, prothrombin time). Hepatic and renal injury become clinically apparent and may progress to fulminant hepatic failure and renal failure. 
Death occurs within 3-7 days or recovery within 2-3 weeks.

\section{Adverse prognostic criteria in acute liver}

\section{failure $^{2}$}

1. Prothrombin time $>100 \mathrm{sec}$

Or

Any three of the following

i) Prothrombin time $>50 \mathrm{sec}$.

ii) S. Bilirubin level: $>300 \mu \mathrm{mol} / \mathrm{L}(\mathrm{H} " 17.6 \mathrm{mg} / \mathrm{dl})$

iii) $\mathrm{S}$. Creatinine :>300 $\mu \mathrm{mol} / \mathrm{L}(\mathrm{H} " 3.38 \mathrm{mg} / \mathrm{dl})$

iv) Jaundice to encephalopathy time $<7$ days

v) Age $<10$ yrs or $>40$ yrs

or

Factor V level of $<15 \%$ of normal and

encephalopathy grade 3 or 4 (marked

confusion/ coma)

These criteria predict a mortality rate of $\geq 90 \%$.

\section{Diagnosis of mushroom poisoning}

1. From clinical features, a positive history of mushroom intake is present. Symptoms may be mild and early, when the mushroom species is less toxic, amount ingested is less and single species is consumed: but when different species of mushrooms are ingested, early symptoms do not exclude delayed and fatal complications of a very toxic species. Very toxic mushroom poisoning cases usually have delayed onset of symptoms, often with hepatic, hemolytic, renal and CNS involvement.

2. If a specimen of the ingested mushroom is found, analysis for amatoxins done by a Maixner test ${ }^{3}$ by expressing a drop of liquid from the specimen on a paper, containing wood pulp e.g. news paper, allowed to dry and placing a drop of 1012 N HCL on this spot. After several minutes, there will be appearance of a blue colour if amatoxin is present.

3. An experienced mycologist may analyse and identify spores in gastric contents. ${ }^{3}$ A regional toxicology centre may be informed.

4. High performance liquid chromatography for detection of amatoxin from plasma, faeces, urine or vomits to be done if facilities are available.

\section{Differential diagnosis}

1. Gastroenteritis

2. Plant poisoning from different species

3. Hypovolumic shock and

4. Other causes of acute liver and renal failure

\section{Management}

1. Pre-hospital care- Institute supportive measures if needed viz. I.V. access and Oxygen and induce vomiting.

If a mushroom sample is available, place it in a dry paper bag (do not moisten or refrigerate) for a mycologist to identify.

2. Emergency department care-aggressively treat a patient with suspected mushroom ingestion as the mortality of ingested amatoxin is very high - about $20 \% .4$

\section{Reduction of amatoxin absorption. ${ }^{4}$}

Consider gastric lavage if the patient has not already vomited. Lavage should be attempted within 1 hour of ingestion. Given the delayed presentation, efficacy of this procedure is uncertain as patient becomes symptomatic and seek medical attention usually after a delay of 12 hours or more.

However, give repeated doses of activated charcoal orally for any recent ingestion of an unidentified or potentially toxic mushroom. Amatoxin appear to 
B. S. Patowary, Mushroom Poisoning-an overview

undergo entero - hepatic circulation, repeat dose activated charcoal and laxatives may interrupt this cycle and reduce toxicity. Gastroduodenal aspiration may be done to remove the toxins eliminated in bile and interrupt this cycle and reduce toxicity.

Mainstay of treatment include aggressive intravenous fluids and electrolytes; to correct and maintain adequate hydration and urinary out put as well as intensive supportive care for hepatic failure.

\section{Laboratory Studies}

- Obtain liver function tests, as hepatic damage is the main concern in Amatoxin poisoning.

-Prothrombin time (PT): most reliable indicator for severity of poisoning.

-Amino transferase levels

-Alkaline Phosphatase level

-Bilirubin level

- $\quad$ Complete blood count, glucose level

- $\quad$ Electrolytes, Blood Urea, S. Creatinine level (dehydration from vomiting, diarrhoea)

- Urine analysis ( Proteinuria and haematuria signify renal involvement)

- $\quad$ Amylase/Lipase level ( Pancreatitis)

- Urinary amanitin analysis (if facilities available)

Antidotes: Several have been proposed, but none have proven to be of clinical efficacy as controlled studies are lacking and experimental data in animals are equivocal. ${ }^{4}$

a) Benzylpenicillin (Penicillin G): Based on the hypothesis that in liver failure due to Amanita, GABA derived from enteric bacteria may be insufficiently metabolized; high dose Penicillin is used to sterilize and reduce the GABA - producing intestinal flora and may prevent the severe encephalopathy likely to be the final cause of death : Dose upto 1 millon U/kg/ day I.V. b) Silibinin/sylimarin: a potent anti-oxidant, prevents free radical damage and amatoxin uptake by liver cells. Early administration (within 48 hours) may be an effective measure. Dose $20 \mathrm{mg} / \mathrm{kg} /$ over 24 hours given in four 2 hours infusions.

c) Thioctic acid-clinical efficacy not yet proven.

d) Cimetidine - it is a cytochrome $\mathrm{P} 450$ inhibitor.

e) Vit.K - if coagulopathy is present

f) N-Acetylcysteine - not definitely proved. It improves cerebral blood flow and oxygenation in patients with fulminant hepatitis due to any cause.

g) Corticosteroids - not proved.

Hemodialysis-to remove mushroom poison early. It has become a part of the treatment programme in the United States along with other schedules.

For fulminant hepatic failure - consult for liver transplantation as liver transplant can save the life ${ }^{4}$ of a patient with most severe amatoxin poisoning.

\section{Criteria for transplantation ${ }^{5}$}

A prolonged Prothrombin time ( a measure of blood clotting)

Elevated levels of liver enzymes (indicative of liver dysfunction)

Increased levels of ammonia in blood.

Other symptoms eg. Hepatic encephalopathy (Gr. II -III)

When the interval between ingestion and the onset of diarrhoea is $<8$ hours

or

The INR is $\geq 6.0$ even in the absence of encephalopathy.

When indicated, the operation should proceed as soon as possible, before the effects of liver failure become more widespread. 


\section{Discussion}

No adequate database exists to estimate the worldwide exposure and fatality due to mushroom poisoning.

Patients with severe hepatitis from mushroom poisoning are thought to have a poor prognosis and frequently need liver transplantation for survival. But with early and aggressive multidisciplinary care, such patients have improved outcome and may avoid liver transplantation, as suggested by Rengstorff et al, $2003 .{ }^{6}$

From San Francisco, USA, from their retrospective study of 8 admitted patients over 5 year period - all the 8 patients survived; even though 3 had developed encephalopathy ( grade I to III), and 1 developed acute renal failure requiring hemodialysis. In another 15 - years retro spective analysis and follow up evaluation of 105 patients from Florence, Italie ( Giannini et al, 2007) ${ }^{7}$ deaths have been recorded only in 2 patients admitted 60 hours after mushroom ingestion, while the rests recovered completely without sequalae and all of them were treated within 36 hours after mushroom ingestion - none of them had liver transplantation. Again, a 20 year retrospective analysis of treatment of amatoxin poisoning in 2108 hospitalized patients from North America and Europe (Enjalbert et al, 2002) ${ }^{8}$ documented little efficacy of Penicillin G, thioctic acid or steroids, they observed that silybin, $\mathrm{N}$-acetyl cysteine and detoxication procedures were efficacious.

\section{Prevention}

Most important factor is not to eat wild or uncultivated mushroom as it is very difficult to correctly identify edible from poisonous mushroom. It is important to remember that most lethal mushroom toxins are not destroyed or deactivated by cooking, canning, freezing, drying or other means of food preparations. It is best to avoid alcohol while consuming mushroom. It is worth remembering that there is no specific antidote for lethal mushroom poisoning.

If there is suspicion of consuming a poisonous mushroom, immediately a physician /hospital to be contacted or to approach a local poison control center for the needful.

Aiming at primary prevention, the government should establish regional toxicology centers which would impart public education on recognition of toxic mushroom along with first aid management.

Every physician should consider amanita toxicity in the differential diagnosis of acute hepatic and renal failure, especially in high prevalent regions.

\section{Acknowledgement}

I offer my thanks to Mr. Ajay Baruah and Mrs. Sumitra Neupane for their timely help with the computer.

\section{References}

1. Jules L D. Toxic and Drug Induced Hepatitis. Dennis L. Kasper, Anthony S. Fauci, Dan L. Longo et al. Harrison's Principles of Internal Medicine: Mc GrawHill. $17^{\text {th }}$ ed. Newyork: McGraw-Hill; 2008: 1949-51.

2. Jones A L, Karalliedde L.Poisoning. Davidson's Principles \& Practice of Medicine. $20^{\text {th }}$ ed. New Delhi: Churchill Livingstone Elsevier; 2006: 952-3.

3. Litovitz T L, Smilkstein M, Felberg L et al. 1996 annual report of the American Association of Poison Control Centers Toxic Exposure Surveillance System. Am J Emerg Med. 1997; 15: 447-500.

4. Kent R. Poisoning. Stephen J, Maxine A. Current Medical Diagnosis \& Treatment. $47^{\text {th }}$ ed. Newyork: McGraw-Hill Lange; 2008: 1378-9. 
B. S. Patowary, Mushroom Poisoning-an overview

5. Pinson C W, Daya M R, Benner K G et al. Liver transplantation for severe Amanita Phalloides mushroom poisoning. Am. J. Surg 1990; 159: 493-9.

6. Rengstorff D S, Osorio R W, Bonacini M. Recovery from severe hepatitis caused by mushroom poisoning without liver transplantation. J. Clin Gastroenterol Hepatol 2003;1:392-6.
7. Giannini L, Vannacci A, Missanelli A et al. Amatoxin poisoning: A 15-year retrospective analysis and followup evaluation of 105 patients. J Clinical toxicology, 2007; 45: 539-42.

8. Enjalbert F, Rapior S, Nouguier Soule et al. Treatment of amatoxin poisoning: 20-year retrospective analysis. J. Clin. Toxicol. 2002; 40: 715-57. 